\title{
The COVID-19 Pandemic and Implications for Gynaecologic Cancer Care
}

\author{
Neerja Bhatla $^{1} \cdot$ Seema Singhal ${ }^{1}$
}

Received: 29 March 2020 / Revised: 6 April 2020 / Accepted: 11 April 2020/Published online: 24 April 2020

(C) Association of Gynecologic Oncologists of India 2020

\begin{abstract}
Purpose The impact of the COVID-19 pandemic on world healthcare system and economy is unprecedented. Currently routine surgical procedures are at a halt globally, but whether one can delay cancer procedures remains an ethical issue, and still there is no clarity on how women with gynaecological cancers should be managed in these critical times.

Methods Currently available literature on impact of COVID-19 on cancer was reviewed with special reference to its applicability to the Indian context.

Results Cancer cases are more susceptible for COVID-19 infection and rapid deterioration if they get infected. A tumour board should plan their management with a "do no harm" approach as the guiding principle. Teleconsultation may be used to advise patients for therapy and symptom control measures, as well as to advise new patients regarding diagnostic tests. Surgical decision making may be stratified into three categories: patients with low (not life threatening) or intermediate (potential for future morbidity or mortality) acuity may be delayed; those with high acuity may be taken up for planned therapy after explaining the risks. Assessment of the severity of disease, comorbid conditions, and logistic challenges, along with COVID census in their area are important variables for informed and individualized decision making. Safety of healthcare personnel needs to be ensured at the same time.

Conclusion Currently available evidence is limited by small sample size, and full impact of this pandemic on cancer is yet to be seen. However, cancer care needs to be individualized taking all variables into consideration.
\end{abstract}

Keywords COVID-19 · Pandemic - Gynaecological cancer care · Coronavirus infection and cancer

\section{Introduction}

In February 2020, COVID-19, caused by the novel SARSCoV-2 (severe acute respiratory syndrome coronavirus-2), was declared as a public health emergency of international concern by the World Health Organization [1]. Since its inception in December 2019, the number of cases is rising exponentially worldwide. Since this is a novel virus, the human population does not have any prior immunity to fight against the disease nor there is any vaccine or therapy available [2]. The median duration of viral shedding is reported to be 20.0 days (IQR 17.0-24.0) [3, 4]. Hence, it is anticipated that the infection will remain at least for

Seema Singhal

drseemasinghal@gmail.com

1 Department of Obstetrics and Gynecology, All India Institute of Medical Sciences, New Delhi, India another 2-3 months before clearing. The impact of COVID-19 is substantial and has caused enormous economic, social and healthcare disruption worldwide. COVID-19 infection has been observed to cause mild illness and recovery in the majority (80\%) of cases, more severe illness is seen in $14 \%$ cases, and only about $6 \%$ cases develop critical illness [5]. The risk of death is higher in those with higher SOFA (Sequential Organ Failure Assessment) score (5.65, 2.61-12.23; $p<0.0001)$, d-dimer $>1 \mu \mathrm{g} / \mathrm{L}(18.42,2.64-128.55 ; p=0.003)$. The impact of the illness is highest in the elderly (odds ratio 1.10 ; 95\% CI 1.03-1.17; $p=0.0043$ ), and those with known comorbid illnesses including cancer [4].

Considering the unprecedented dynamic situation and uncertain epidemiological trends, new policies and recommendations continue to evolve on a daily basis. Currently routine surgical procedures are almost at a halt worldwide, but whether cancer procedures can be delayed 
remains an ethical issue. Most of the advisories do not recommend stoppage of care for cancer patients for low COVID census areas [6]. However, the current unprecedented lockdown enforced to reduce community transmission poses an ethical challenge to gynaecologic oncologists as there is insufficient evidence to determine the impact of this novel infection on cancer care and therefore no clarity on how women with gynaecological cancers should be managed in this critical time.

The present article explores the impact of the virus on cancer and of cancer on the management of infected patients and suggests possible options for providing optimum care.

\section{Impact of Cancer on COVID-19 Infection}

There is limited information till date about the impact of cancer on COVID-19 patients; however, cancer patients are at increased risk of COVID infection due to their systemic immunosuppressive state. The only paper published is a nation-wide analysis from China, in which only 18 (1\%) out of 1590 COVID positive cases had a history of cancer [7]. Of these, six were women, but there was no case of gynaecological cancer-three had carcinoma breast, one had thyroid cancer and two had lung cancer. Overall, 5 (18\%) of the cancer cases had carcinoma lung. The treatment history of two cases was not known, $25 \%$ (4/16) had received either surgery or chemotherapy in the past 1 month, and the remaining 12 cases were cancer survivors on routine follow-up. It was observed that patients with cancer were older (mean age 63.1 years vs. 48.7 years), had more tachypnoea (47\% vs. $23 \%$ ) and had more severe baseline findings on chest computed tomography ( $94 \%$ vs. $71 \%$ ). There was no difference in gender, other baseline symptoms, comorbidities or baseline severity of chest radiographs. Risk of severe events defined as intensive care unit (ICU) admissions, need for mechanical ventilation or death was higher in cancer patients than in the general population (39\% vs. $8 \% ; p=0.0003$ ). History of cancer was found to be the most significant risk factor for severe events after adjusting for other risk factors including age, comorbidities and history of smoking (odds ratio 5.34, 95\% CI $1.80-16.18 ; p=0.0026)$. Similarly, the risk of rapid deterioration was also greater in those with history of cancer (HR 3.56; 95\% CI 1.65-7.69). Patients who underwent surgery or chemotherapy in the past 1 month had a higher risk of severe events ( $75 \%$ vs. $43 \%)$. Patients with history of lung cancer did not have an increased probability of deterioration [7]. The study emphasized that cancer patients are particularly at risk for adverse outcome and rapid deterioration because of their systemic immunosuppressive state due to malignancy, previous recent or ongoing therapy.

A second report from China has retrospectively analysed the clinical features, treatment and outcome of three COVID-19 positive women with gynaecological cancers. Pneumonia was cured in two cases, and one woman died of COVID illness. It was reiterated that women with gynaecological malignancies are susceptible for COVID-19 infection and rapid deterioration [8].

Considering the susceptibility of cancer patients to severe coronavirus infection, the increased likelihood of ICU admission and need for mechanical ventilation if they deteriorate, and eventually poorer outcomes, all cancer patients should be informed of the need for stronger personal protection measures like social distancing, use of personal protective equipment, isolation, etc.

\section{Impact of COVID-19 Infection on Cancer}

The potential threat of COVID-19 to women with gynaecological cancers is substantial. In the current scenario, there will be delays in diagnosis, seeking treatment and actual treatment administration. Treatment delay is known to be an independent risk factor for increased mortality due to cancer. According to a study in Taiwan, the survival of women with cervical cancer (all stages) at 1 and 5 years was significantly worse for those who did not receive treatment within 4 months of diagnosis in comparison with those who received timely therapy after adjusting for other known risk factors. (1-year survival: $91 \%$ vs. $60 \%$; 5-year survival: $71 \%$ vs. $38 \%$ ) [9].

Shalowitz et al. [10] investigated the impact of timing from diagnosis of endometrial cancer to surgery on survival. It was observed that for both low- and high-risk endometrial cancer, the 5-year survival was in fact better if surgery was done in the third week after diagnosis compared to week one or two (low-risk disease: $87.4 \%$; highrisk disease $66.9 \%$ ). For low-risk endometrial cancer, the risk of death increased if surgery was conducted after the 8th week of diagnosis. Subsequently, the 5-year survival was $16 \%$ inferior for cases who underwent surgery after 4 months of diagnosis than for patients who underwent surgery within 3 weeks. Conversely, for high-risk disease, a delay of up to 21 weeks did not have a significant impact on survival in this study [10]. Interestingly, rapid surgery within the first week of diagnosis also had an adverse impact on survival $[10,11]$.

The majority of ovarian malignancies are diagnosed in advanced stages, and further delay may have a detrimental effect on the outcome. However, the timing of initiation of adjuvant chemotherapy also has a direct impact on survival. According to a meta-analysis by Liu et al. [12], the 
overall survival was worse in women in whom administration of adjuvant chemotherapy was delayed more than 6 weeks (HR 1.18, 95\% CI 1.06-1.32). Hence, on the one hand if delay in therapy is detrimental, the risk of ICU admission, mechanical ventilation and death is 3.5 times more if they acquire COVID-19 infection. These factors have to be taken into consideration in the decision on whether to treat immediately or delay the definitive surgery; if surgery is done, it needs to be ascertained whether it will be feasible to administer adjuvant therapy within 3-6 weeks of surgery as per the standard practice. Counselling patients, allaying their anxiety and calming the fear of delay in therapy versus the risk of therapy in the current compromised healthcare scenario is another challenge that needs to be addressed on an individual basis. Moreover, the reality of exhausted supplies and limited resources is another unprecedented situation [3, 4]. Availability of ICU beds and ventilators is a real challenge presently. It is speculated that channelling diagnostic, therapeutic (surgical, medical or radiation oncology) and ICU resources for care of cancer cases will be in direct conflict with COVID19 services as this pandemic progress, but this has ethical concerns as well. Safety of healthcare workers and limited availability of COVID-19 testing along with judicious use of PPE need further attention. The major concern for cancer patients now is getting admitted in hospital and receiving definitive therapy after admission which may be delayed or denied owing to resource diversion [13].

\section{Impact of COVID-19 on the Pre-existing Burden of Gynaecological Cancers in India}

Gynaecological cancers are an important public health problem in India with 155,074 new cases and 93,777 deaths every year. According to GLOBOCAN estimates, there were 96,922 new cases of cervical cancer, 13,328 new cases of endometrial and 36,170 new cases of ovarian

Table 1 Stage-wise distribution of gynaecological cancers in India [17-21]

\begin{tabular}{lllll}
\hline Site & Stage I & Stage II & Stage III & Stage IV \\
\hline Cervix & IA-11\% & II A-5\% & III A-1\% & \\
& & II B-30\% & III B-53\% & \\
Endometrium & I A-34.5\% & $7.2 \%$ & $25.5 \%$ & $5.45 \%$ \\
& I B- 27.7\% & & & \\
Ovary & & $20 \%$ & $60 \%$ & \\
Vulva & $3.3 \%$ & $28.3 \%$ & $51.6 \%$ & $15 \%$ \\
Vagina & $6.6 \%$ & $69.3 \%$ & $18.6 \%$ & $5.3 \%$ \\
\hline
\end{tabular}

cancer in India in 2018 [14]. Approximately 70\% of gynaecological cancer cases present in advanced stages (Table 1), around $20 \%$ default during the preliminary work-up and another $30 \%$ default after starting adjuvant therapy. These factors result in poor survival and high mortality rates [15]. The disease stage remains the most important prognostic variable affecting survival [16]. The 5-year DFS was $56 \%$ in women with cervical cancer when they were diagnosed in stage IIb, but it reduced to $40 \%$ when the disease was more advanced [17]. Similarly, the 5-year OS in ovarian cancer is reduced to $30 \%$ and $15 \%$ when the disease is diagnosed in stage III and stage IV, respectively, as compared to stage II when survival is as good as $65 \%$ [18]. The patients with endometrial cancer who present with advanced stage were at higher risk of relapse (HR 5.78; 95\% CI 1.18-28.57) and death (HR 6.89; 95\% CI 1.76-27.02) [19]. Information about the stage-wise distribution of gynaecological cancers in India is presented in Table 1. Further delay in providing care in the current situation is likely to upstage the disease and increase the burden of more advanced, inoperable or incompletely treated cases on cancer care services.

\section{Surgical Decision Making and Stratification Based on Risk-Benefit Analysis}

Surgery in cancer cases is time sensitive. The decision to perform or postpone surgery should be made based on the type and stage of the disease, medical condition of the patient, area census of COVID-19 cases, COVID-associated risks, and available logistic support including adjuvant treatment services [6]. In order to carry out surgical procedures in an orderly and safe manner, an acuity scale is suggested to guide gynaecologic oncologists whether to postpone or perform surgical procedures during the COVID-19 pandemic in the current phase in India (Table 2).

For pre-invasive lesions and slow growing cancers, it may be prudent to postpone the surgery. Cases with comorbidities, as seen in the case of endometrial cancer, may be advised to wait till their condition is optimized and not rush into surgery. Laparoscopy is not being recommended mainly due to the probable effects of pneumoperitoneum; hence, such cases are better postponed [22]. Surgery that is unlikely to require adjuvant treatment may be a better case for consideration; however, cases that are likely to require ICU care are also best postponed as these facilities may be reserved for COVID-19 positive cases and limited or unavailable for routine cancer cases. In the case of advanced cancer and treatment with palliative intent, surgery is best postponed. They may be considered 
Table 2 The suggested acuity scale for surgical decision making in hospitals with low or no COVID census (adapted from American College of Surgeons COVID-19 recommendations for management of elective surgical procedures)

\begin{tabular}{|c|c|c|c|}
\hline Description & Definition & Examples & Suggested plan \\
\hline Category 1 & $\begin{array}{l}\text { Low acuity surgery } \\
\text { (Not life threatening) }\end{array}$ & $\begin{array}{l}\text { Treatment of pre-invasive lesions of cervix or } \\
\text { endometrium }\end{array}$ & $\begin{array}{l}\text { Postpone surgery for few weeks or months } \\
\text { e.g. conization to rule out invasion may be } \\
\text { delayed for weeks but for HSIL may be } \\
\text { delayed for months }\end{array}$ \\
\hline Category 2 & $\begin{array}{l}\text { Intermediate acuity } \\
\text { surgery } \\
\text { (Not life threatening but } \\
\text { potential for future } \\
\text { morbidity and mortality) }\end{array}$ & $\begin{array}{l}\text { Low-risk cancer } \\
\text { (e.g. early cervical cancer, well differentiated } \\
\text { endometrial cancer with comorbidities) }\end{array}$ & $\begin{array}{l}\text { Postpone surgery if possible after informed } \\
\text { decision making or consider early } \\
\text { discharge. } \\
\text { Cases with comorbidities should preferably } \\
\text { be counselled for rescheduling surgery }\end{array}$ \\
\hline Category 3 & $\begin{array}{l}\text { High acuity surgery/ } \\
\text { healthy patient } \\
\text { (Life threatening) }\end{array}$ & $\begin{array}{l}\text { Most cancers, highly symptomatic patients } \\
\text { (Type II endometrial cancers, ovarian cancer, interval } \\
\text { debulking surgery after 3-4 cycles of chemotherapy, } \\
\text { uterine sarcoma, those in need of emergency } \\
\text { procedures, excision of malignant recurrences, } \\
\text { GTN) }\end{array}$ & $\begin{array}{l}\text { Do not postpone if COVID census low and } \\
\text { resources permit. } \\
\text { A multidisciplinary team discussion and } \\
\text { planning of therapy is preferred prior to } \\
\text { surgery for realistic expectations. }\end{array}$ \\
\hline
\end{tabular}

Special circumstances

If healthcare facilities are burdened by many COVID-19 cases with accelerating graphs and limited supplies being available, only life-saving procedures may be done and consider observation for all. Alternatively neoadjuvant therapy may be considered for carefully selected eligible cases after informed consent

However, in situations where all hospital resources are routed to COVID 19 patients, with limited or no ICU, HDU capacity or no mechanical ventilators being available all cases should be deferred

It is safe to donate blood and therefore donors should be encouraged to maintain constant supply

Laparoscopic procedures are preferably avoided

for chemotherapy and palliative management as appropriate.

\section{Delivery of Services During COVID-19 Pandemic}

\section{Initial Preparedness of Gynae Oncological Care Centres}

The basic principle of medicine_- "Primum non nocereFirst do no harm" is to be recapitulated. Patients and caregivers should not be exposed to additional risks, so also the safety of medical personnel should be ensured. It is the duty of the gynae oncologist to carry out education, prevention, control and treatment of patients according to their condition. Patient information leaflets directing about COVID-19 disease, its course and infection prevention techniques should be developed in local language. Patient help lines may be developed to guide them appropriately. The strategy for promoting "Stay at Home" should be reinforced [23]. Use of personnel protection remedies and social isolation should be reinforced for cancer patients to reduce the probability of getting infection [24].
Safety of health personnel is equally important. Until such time that COVID testing becomes widely available, every patient should be considered a potentially infected case and a policy of universal precautions should apply. Personal protective equipment (PPE) for all staff members, along with screening facility for those involved in patient care, should be promoted. DO's and DON'Ts for healthcare professionals should be liberally displayed. The facility to quarantine exposed healthcare professionals should be designated. Medical teams should be divided into three groups working for 1 week at a time. This will ensure that in the case of exposure, only one-third of the staff need to be isolated/quarantined and this will not impact services. It should also be noted that in case a health professional acquires the infection, there is enormous potential for spread to other patients. Hence it is desirable that services be curtailed judiciously, and the minimum number of persons be brought into the team. Virtual meetings and consultations should be carried out wherever possible.

\section{Preventive Services}

Women who are due for any kind of screening test should be advised to reschedule the visit once the pandemic gets settled and reassured that a delay of a few months will not 
have a significant impact. Similarly, those due for treatment or follow-up after treatment of pre-invasive lesions may also delay their visit for a similar period. They should be advised to consult the facility telephonically in case they have any symptoms, in which case diagnostic procedures may be advised.

\section{Diagnostic Services}

All urgent diagnostic procedures including biopsy, endoscopies, paracentesis or routine work-up for surgery should be performed in outpatient settings. Facility for disinfection of the unit should be available if a suspected or confirmed case is taken for imaging. Wherever possible, home-based diagnostic services, e.g. blood sample collection, portable ultrasonography, etc., should be offered to elderly patients. However, in an area with high COVID incidence, elective diagnostic services may be postponed after discussing the pros and cons and clear documentation.

\section{Outpatient Care}

Patients may either be undergoing diagnostic evaluation to confirm diagnosis, waiting to start therapy after confirmation, or already undergoing therapy. In whatever phase of management they may be, it is important to avoid visiting hospital unless there is an emergency or a clear plan of management that requires a visit (Fig. 1). Only patients who need urgent care should be seen in hospital. They should be advised to bring only one attendant with them. All others may either be provided teleconsultation with clear documentation or may be rescheduled for a future appointment. In the current scenario of complete lockdown and social distancing, teleconsultation is the safest and most feasible option for all patients. It has been permitted by the Medical Council of India and guidelines are available [25]. It is preferable to use tablet computers. Ensuring eye-to-eye contact with the patient reinforces empathy and concern for the patient during teleconsultation [25]. Patients under surveillance after completion of therapy should schedule follow-up visits after the pandemic is over and meanwhile report only if there are any significant symptoms.

\section{Triage of Cases}

Triage of all cases and directing suspects with fever, respiratory and or gastro-intestinal symptoms to the respective designated area is vital to curtail risk of exposure to staff and other patients. These suspects can then be considered for COVID-19 screening after consulting the specialists as per local policy [24].

\section{Approach for Optimum Care}

A multidisciplinary team consisting of gynaecologic oncologist, medical oncologist, radiation oncologist, psycho-oncologist and palliative care specialist should plan the management of women with gynaecological cancers. An intensivist should be included in the team for suspected COVID-19 cases. The realistic timelines for multimodality

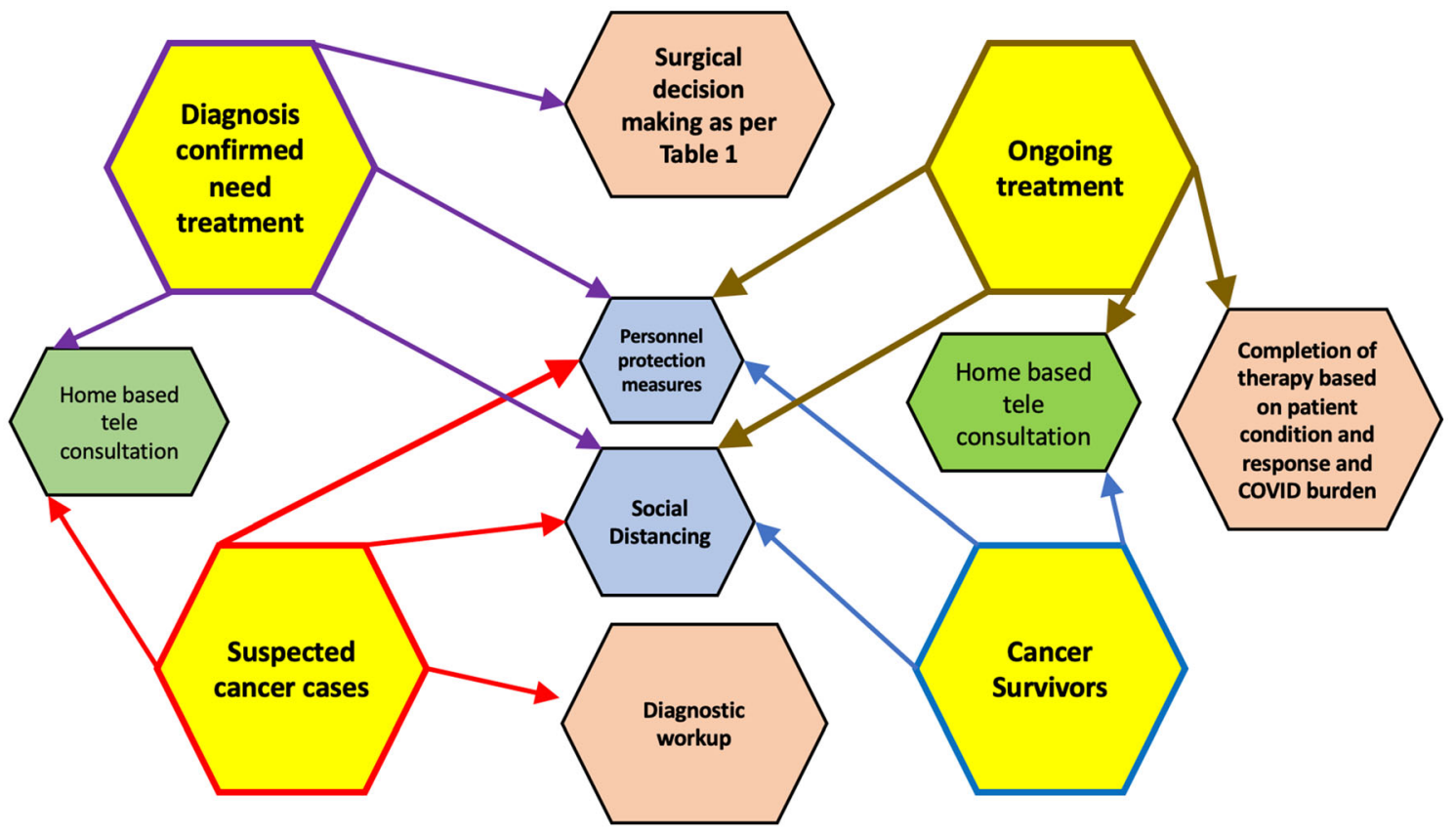

Fig. 1 Suggested algorithm for management of women with gynaecological cancers during COVID-19 epidemic 
treatment need to be discussed, and an informed written consent should be taken explaining the impact of possible delay, treatment interruptions on disease outcome and also the possibility of rapid deterioration in case they acquire COVID-19 infection. A team leader should be identified, and regular virtual meetings should take place for continuous appraisal/audits and planning to combat the crisis. It is preferable that all known or suspect cancer cases should be segregated and managed in high dependency or ICU areas as per their medical needs [24]. It is important to emphasize prophylactic measures like limiting visitors to hospitals or self-quarantine [2, 22, 24].

\section{Chemotherapy and Radiation Therapy}

Whether to start chemotherapy or postpone for another 6-8 weeks after surgery is another issue that remains unanswered due to lack of specific evidence and guidelines. Considering the findings of the Chinese study, these patients should also be explained the risk of increased susceptibility to COVID-19 infection, higher risk of chemotherapy-induced adverse effects, and possibility of faster deterioration in case they acquire the COVID-19 infection. They and their families must understand the impact of delay on cancer outcome and healthcare limitations to provide optimum care. Thus each case should be individualized and treatment planned accordingly. For newly diagnosed advanced cases, neoadjuvant chemotherapy (NACT) may be considered. Those who are already receiving NACT may continue, and if logistics do not permit performance of surgery after 3-4 cycles, all six cycles may be completed [22, 24]. However, in situations with increasing number of cases cytotoxic chemotherapy may also be postponed.

Initiation of radiation therapy within 6 weeks of surgery is also an important prognostic factor, but considering the lack of recommendations during this crisis, one may individualize its use and reschedule accordingly. Hypofractionation is an option that will reduce the number of hospital visits. Brachytherapy may be carried out as per schedule for non-COVID cases [22, 24].

Patients needing palliative care are better managed at home. Adequate supply of drugs and other supplies should be ensured and helplines provided so that required support may be provided telephonically.

\section{Current COVID Crisis}

Figure 1 summarizes the suggested algorithms for administering gynae cancer care to patients during COVID pandemic. The following suggestions may be considered during administration of care to gynaecologic oncology patients during the current COVID-19 pandemic in India:

- If resources permit, surgical decision making may be done as per Table 2; otherwise elective procedures may be minimized, postpone or cancelled until the predicted infection point in the exposure graph is crossed.

- Holistic assessment of the risk of performing immediate surgery versus the risk of delaying the procedure to emerge from the COVID-19 crisis should be made by an experienced multidisciplinary team.

- Many asymptomatic individuals who are in the carrier stage are unknowingly exposing other patients and healthcare professionals. The use of essential items including PPE and cleaning supplies needs to be optimized by appropriate organization of teams. The available evidence highlighting the increased susceptibility and poor outcome of cancer patients is limited by small sample size and lack of generalizability and therefore insufficient to recommend a specific advisory. Data should be pooled to allow quicker analysis.

\section{Plan of Management If the COVID Crisis Continues and Lockdown is Extended Further}

If the situation arises that the lockdown needs to be extended, cancer care services may have to be resumed in selected places. In an ideal setting, it would be preferred that all potential surgical candidates are screened for COVID infection, but screening protocols may have to be tailored depending on resources and local guidelines and universal precautions may need to be followed. The following practical points may be considered while considering reinstallation of cancer care services if the COVID crisis continues in India:

1. Since it is preferred that women with suspected cervical cancer should ideally receive diagnostic evaluation within 4 weeks from the initial presentation, such cases may be scheduled for diagnostic conization/ LEEP (loop electrosurgical excision) after careful counselling and evaluation. Those with high grade lesions on cytology screening may wait up to 3 months and those with low grade lesions may be counselled for following up after the crisis is resolved (6-12 months) [26].

2. Women with early cervical cancer who have been waiting for the last 6-8 weeks should be re-evaluated for operability and COVID census and resources. Definitive surgery is preferred if resources permit; 
otherwise NACT may be considered in situations with ongoing crises beyond 8 weeks.

3. In women with low-risk uterine cancer, who have been waiting or expected to delay beyond 6-8 weeks, a nonsurgical option may be considered after careful selection of cases. Conversely, high-risk cases may be taken for surgery on priority even in extended lockdown situation. In cases with advanced or recurrent disease, a confirmatory biopsy may be taken, and systemic therapy or hormonal therapy may be considered.

4. In an extended lock down situation and ongoing crisis, women with ovarian cancer should be carefully counselled and continue with NACT and may complete all 6 cycles, but if surgical services can be resumed, they should be considered as priority. However, the impact of chemotherapy on immunity and increased susceptibility to COVID infection remain a concern. If possible, a COVID screen may be done prior to surgery for such cases. Those with yolk sac tumours should be provided surgical staging and chemotherapy at the earliest possible time because of higher risk of disease progression and compromised outcome.

5. For rare cancers like vulva and vagina, neoadjuvant chemoradiation may be considered.

\section{Re-starting Routine Care After Resolution of COVID Crises}

Once the situation improves and the hospital resumes routine services, the availability of hospital supplies will need to be ensured before scheduling patients for therapy. It will be prudent for clinicians to strike a balance between already waiting cases and new arrivals. However, decision making will need to be tailored according to the individual patient needs and circumstances at each facility. There will be a backlog of ovarian cancer cases who are currently receiving NACT and need interval cytoreduction on priority. Likewise, patients with cervical malignancy who are found operable on re-evaluation will be a priority. Resumption of preventive services might further be postponed for another 3-4 weeks till replenishment of resources and healthcare professionals is ensured and services are streamlined.

\section{Conclusion}

The novel coronavirus outbreak is a critical challenge for humanity. The full impact of this pandemic on cancer care is yet to be seen. As the number of cases spread across the country, gynaecologic oncologists should follow a principled, conflict-sensitive "do no harm" approach and continue to provide balanced, compassionate and safe care to patients. Owing to the uncertainties of the current lockdown and number of positive cases, the risk-benefit analysis remains dynamic and is derived by available data on disease magnitude and individual needs.

Funding None.

\section{Compliance with Ethical Standards}

Conflict of interest The authors declare that there is no conflict of interest.

Consent for Publication Taken from all authors.

\section{References}

1. World Health Organization. Coronavirus disease 2019 (COVID19): situation report, p. 51.

2. World Health Organization. Clinical management of severe acute respiratory infection (SARI) when COVID-19 disease is suspected: interim guidance, 13 March 2020. World Health Organization; 2020.

3. Huang C, Wang Y, Li X, Ren L, Zhao J, Hu Y, et al. Clinical features of patients infected with 2019 novel coronavirus in Wuhan, China. Lancet. 2020;395(10223):497-506. https://doi. org/10.1016/S0140-6736(20)30183-5.

4. Zhou FD, Yu T, Du R, Fan G, Liu Y, Liu Z, et al. Clinical course and risk factors for mortality of adult inpatients with COVID-19 in Wuhan, China: a retrospective study. Lancet. 2020. https://doi. org/10.1016/S0140-6736(20)30566-3.

5. Team NCPERE. Vital surveillances: the epidemiological characteristics of an outbreak of 2019 novel coronavirus diseases (COVID-19)—China. China CDC Weekly. 2020;2(8):113-22.

6. Burki TK. Cancer care in the time of COVID-19. Lancet Oncol. 2020. https://doi.org/10.1016/S1470-2045(20)30201-1.

7. Liang W, Guan W, Chen R, Wang W, Li J, Xu K, et al. Cancer patients in SARS-CoV-2 infection: a nationwide analysis in China. Lancet Oncol. 2020;21(3):335-7 (Old 9).

8. Zhang J, Peng P, Li X, Zha YF, Zhang GN, Zhang Y, et al. Management strategies for patients with gynecological malignancies during the outbreak of COVID19. Zhonghua Fu Chan Ke Za Zhi. 2020;55:E011.

9. Shen SC, Hung YC, Kung PT, Yang WH, Wang YH, Tsai WC. Factors involved in the delay of treatment initiation for cervical cancer patients: a nationwide population-based study. Medicine. 2016;95(33):e4568.

10. Shalowitz DI, Epstein AJ, Buckingham L, Ko EM, Giuntoli RL II. Survival implications of time to surgical treatment of endometrial cancers. Am J Obstet Gynecol. 2017;216(3):268-e1.

11. Elit LM, O'Leary EM, Pond GR, Seow H-Y. Impact of wait times on survival for women with uterine cancer. J Clin Oncol. 2014;32:27-33.

12. Liu Y, Zhang T, Wu Q, Jiao Y, Gong T, Ma X, et al. Relationship between initiation time of adjuvant chemotherapy and survival in ovarian cancer patients: a dose-response meta-analysis of cohort studies. Sci Rep. 2017;7(1):1-8. 
13. Wang H, Zhang L. Risk of COVID-19 for patients with cancer. Lancet Oncol. 2020. https://doi.org/10.1016/S14702045(20)30149-2.

14. Bray F, Ferlay J, Soerjomataram I, Siegel RL, Torre LA, Jemal A. Global cancer statistics 2018: GLOBOCAN estimates of incidence and mortality worldwide for 36 cancers in 185 countries. CA Cancer J Clin. 2018;68:394-424.

15. Devi KU. Current status of gynecological cancer care in India. J Gynecol Oncol. 2009;20(2):77-80.

16. Maheshwari A, Kumar N, Mahantshetty U. Gynecological cancers: a summary of published Indian data. South Asian J Can. 2016;5(3):112-20.

17. Shrivastava S, Mahantshetty U, Engineer R, Tongaonkar H, Kulkarni J, Dinshaw K. Treatment and outcome in cancer cervix patients treated between 1979 and 1994: a single institutional experience. J Cancer Res Ther. 2013;9:672-9.

18. Mondal SK, Banyopadhyay R, Nag DR, Roychowdhury S, Mondal PK, Sinha SK. Histologic pattern, bilaterality and clinical evaluation of 957 ovarian neoplasms: a 10-year study in a tertiary hospital of Eastern India. J Can Res Ther. 2011;7:433-7.

19. Dessai SB, Adrash D, Geetha M, Arvind S, Bipin J, Nayanar S, et al. Pattern of care in operable endometrial cancer treated at a rural-based tertiary care cancer center. Indian $\mathrm{J}$ Cancer. 2016;53(3):416.

20. Sharma DN, Rath GK, Kumar S, Bhatla N, Julka PK, Sahai P. Treatment outcome of patients with carcinoma of vulva: experience from a tertiary cancer center of India. J Cancer Res Ther. 2010;6:503-7.
21. Pingley S, Shrivastava SK, Sarin R, Agarwal JP, Laskar S, Deshpande DD, et al. Primary carcinoma of the vagina: Tata Memorial Hospital experience. Int J Radiat Oncol Biol Phys. 2000;46:101-8.

22. Ramirez PT, Chiva L, Eriksson AGZ, Frumovitz M, Fagotti A, Martin AG, et al. COVID-19 global pandemic: options for management of gynecologic cancers. Int J Gynecol Cancer. 2020. https://doi.org/10.1136/ijgc-2020-001419.

23. Novel Coronavirus. Prevention and treatment. https://www.cdc. gov/coronavirus/2019-ncov/about/prevention-treatment.html. Accessed 25 Mar 2020.

24. Ueda M, Martins R, Hendrie PC, McDonnell T, Crews JR, Wong TL, et al. Managing cancer care during the COVID-19 pandemic: agility and collaboration toward a common goal. J Nat Compr Can Netw. 2020;1(aop):1-4.

25. Telemedicine practice guidelines: enabling registered medical practitioners to provide healthcare using telemedicine. https:// www.mohfw.gov.in > pdf > Telemedicine. Accessed 28 Mar 2020

26. ASCCP interim guidance for timimg of diagnostic and treatment procedures for patients with abnormal cervical screening tests. COVID-19-ASCCP. https://www.asccp.org > covid-19. Accessed 6 Apr 2020.

Publisher's Note Springer Nature remains neutral with regard to jurisdictional claims in published maps and institutional affiliations. 